\title{
Facial reconstruction with a unique osteomyocutaneous DCIA perforator flap variant: a case report
}

\author{
G. Wechselberger $\cdot$ K. Schwaiger $\cdot$ J. Hachleitner . \\ G. Oberascher $\cdot$ F. Ensat $\cdot$ L. Larcher
}

Received: 23 June 2015 / Accepted: 19 August 2015 / Published online: 15 October 2015

(C) Springer-Verlag Wien 2015

\begin{abstract}
Summary
Background Anatomical variance of the deep circumflex iliac artery is of high clinical value in facial reconstruction using a deep circumflex iliac artery perforator (DCIAP) flap.

Methods We present the case of a 76-year-old woman treated with an osteomyocutaneous DCIAP flap variant for facial reconstruction. We also review here the literature on DCIA perforator flaps and the different anatomical variances, which might bring clinical benefits.

Results The observed anatomy in our patient offered the possibility to raise a free flap variant with high mobility of a large skin paddle and a long vascular pedicle combined with a variable osteomuscular portion.
\end{abstract}

The case was submitted and presented at the annual congress of the German and Austrian Society of Plastic, Aesthetic and Reconstructive Surgery in Munich, Germany, Sept. 2014 and at the Bozner Symposium of Plastic and Reconstructive Surgery in Jan. 2015 (Bolzano, Italy).

Prof. G. Wechselberger, MD, MAS, MSc $(\bowtie) \cdot$ K. Schwaiger, MD F. Ensat, MD · L. Larcher, MD, MA

Department of Plastic, Aesthetic and Reconstructive Surgery, Hospital of the Barmherzige Brüder Salzburg and Academic

Teaching Hospital of the Paracelsus Medical University Salzburg, Kajetanerplatz 1,

5010 Salzburg, Austria

e-mail: gottfried.wechselberger@bbsalz.at

J. Hachleitner, MD

Department of Oral and Maxillofacial Surgery, Paracelsus Medical

University Salzburg, University Hospital Salzburg,

Salzburg, Austria

G. Oberascher, MD

Department of Otorhinolaryngology-Head and Neck Surgery,

Paracelsus Medical University Salzburg, University Hospital

Salzburg,

Salzburg, Austria
Conclusion The characteristics of the flap thus raised help overcome the disadvantages of the conventional DCIAP flap and offer excellent options for facial reconstruction.

Keywords Iliac flap - Facial reconstruction - DCIAP flap - Groin region - Deep circumflex iliac artery . Ascending branch of deep circumflex iliac artery

\section{Introduction}

Taylor et al. [1] first described the possibility of raising a free flap in the groin region, consisting of an osteomuscular section and the overlying skin, nourished by the deep vascular system (deep circumflex iliac artery perforator flap, DCIAP flap). Compared to the superficial circumflex iliac artery perforator flap (SCIP flap), the DCIAP flap is superior in providing nourishment to the bone and adjacent muscle $[1,2]$. Dependable vascular supply to the osteomyocutanous complex is one of the reasons why the DCIAP flap is a good option in reconstructive surgery involving, in particular, composite mandibular, maxillary, and facial defects.

One of the main problems we encountered while harvesting a DCIAP flap including a vascularized iliac crest as a compound flap was raising the overlying skin island which is attached without great mobility to the bone. The overlying skin is also often limited in size and thus does not provide adequate soft tissue coverage of the defect, which should be treated.

In 1993, Koshima et al. [3] introduced the chimeric composite flaps, consisting of two or more flaps, connected by microanastomoses. These flaps offered greater mobility and variable skin size and thus represented a satisfactory solution. However, this technique is highly complex, time consuming, and due to the several microanastomoses required, highly risky. 
In the case described below, it was possible to achieve complex reconstruction with restoration of the upper jaw and partial bony restoration of the midface and orbit as well as coverage of a huge soft tissue defect with a single flap.

\section{Case report}

A 76-year-old woman presented to our department, requiring facial reconstruction following radical resection of a widespread basal cell carcinoma, involving the right orbit, the anterior base of the skull with infiltration of the dura mater, the cheek, the whole nose, upper lip, right maxilla, the pterygopalatine fossa, and the right epipharynx (Figs. 1 and 2). The patient had a longstanding history of local tumor recurrence, treated surgically several times since 2000 .

One week after resection, reconstruction of the midface was initiated. Reconstruction started after histological confirmation of R0 resection. Our primary intention was to raise a conventional osteomusculocutaneous DCIAP flap as described by Taylor et al. [1] and if necessary to perform a flap supercharging by including and anastomosing the superficial circumflex iliac artery (SCIA) to increase the size of the soft tissue flap. General anatomy of the perforators of the deep circumflex iliac artery (DCIA) and of the SCIA was confirmed using Doppler ultrasonography.

But intraoperative preparation of the proximal DCIA vessels revealed a very large ascending branch about $1 \mathrm{~cm}$ medial to the anterior superior iliac spine (ASIS). Careful surgical preparation and reevaluation with Doppler ultrasonography confirmed the presence of a skin paddle, $15 \mathrm{~cm}$ long, and about $10 \mathrm{~cm}$ wide, superolaterally to the ASIS, which was vascularized by a perforator originating from this ascending branch. Due to this large ascending branch with its large perforator, flap supercharging was not necessary anymore and decision was made to raise this skin paddle together with about $10 \times 4 \mathrm{~cm}$ osseous flap from the full thickness of iliac crest along with the adherent internal oblique muscle. The osteomuscular portion was vascularized by perforators, originating from the deep circumflex iliac artery itself. Due to the superolateral location of the skin paddle, it was possible to mobilize a $15 \mathrm{~cm}$ vascular pedicle together with the bone and muscle, located proximally(Figs. 3 and 6).

After preparation and flap harvest at the donor site, reconstruction and defect closure started. At the donor site, primary wound closure was achieved without complications.

The osteomuscular section was used for reconstruction of the zygomaticomaxillary complex. Fixation was carried out with miniplate osteosynthesis. Intraoral soft tissue reconstruction was performed, using the internal oblique muscle, to induce secondary epithelization. The vessels of the transplant were anastomosed to the facial artery and vein. The huge epidermal defect, also involv-

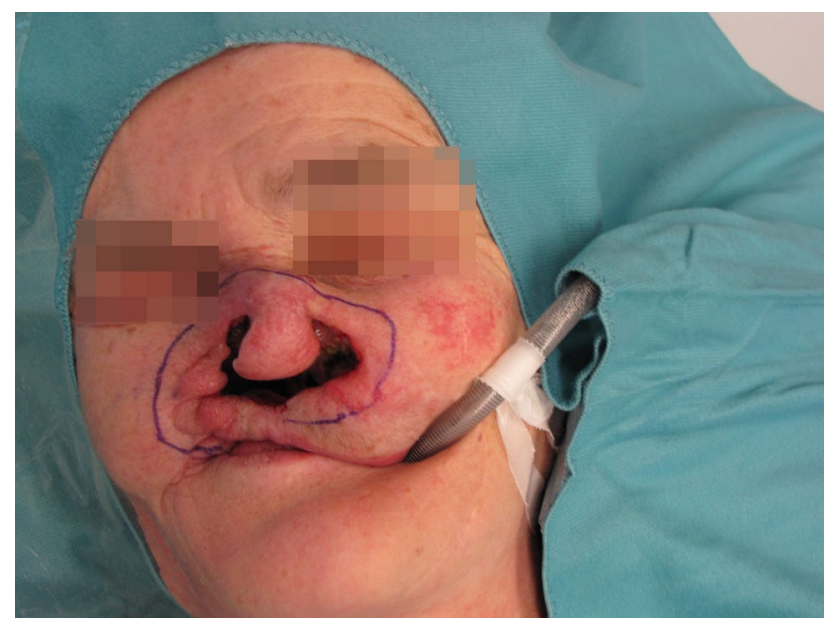

Fig. 1 Preoperative view, a 76-year-old woman with widespread basal cell carcinoma involving the whole right and central midface

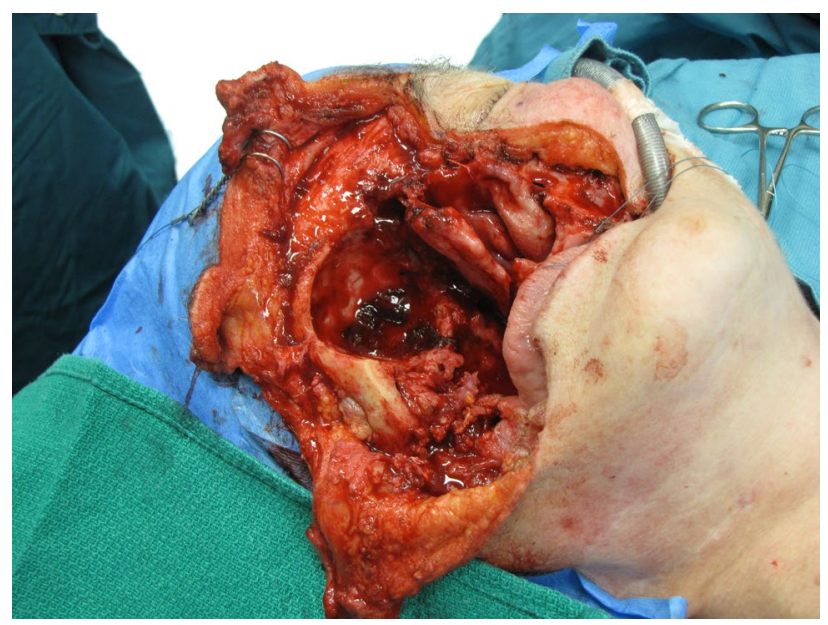

Fig. 2 Intraoperative photo during radical resection. A 76-year-old woman with widespread basal cell carcinoma involving the whole right and central midface

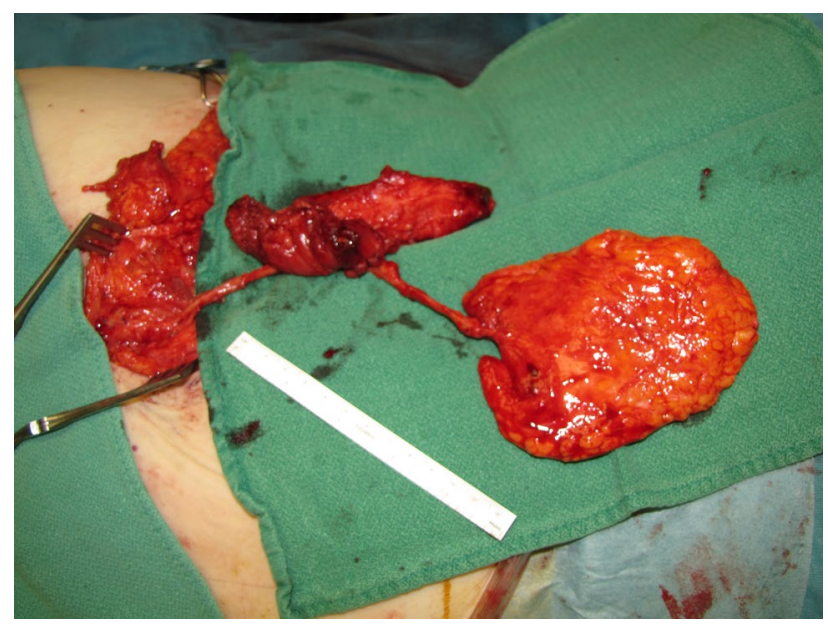

Fig. 3 The DCIA perforator flap variant shown intraoperatively, with its long vascular pedicle connecting the osteomyocutanous part and the skin paddle 


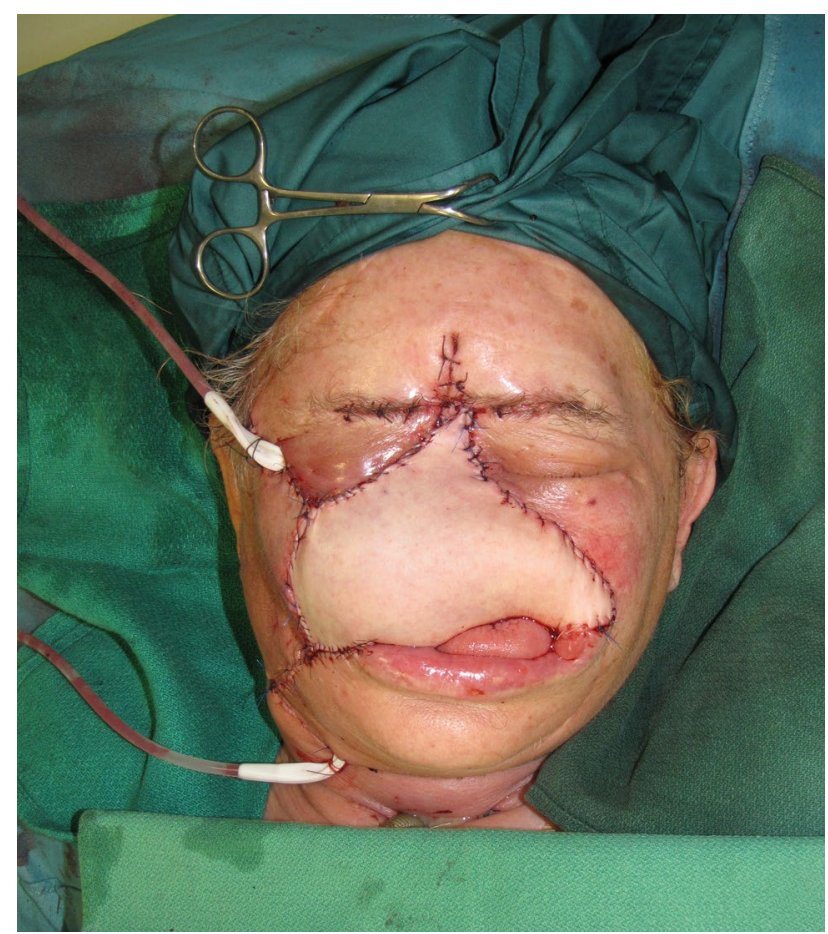

Fig. 4 The patient immediately postoperatively

ing the right orbit due to loss of the eye, was covered with the highly mobile skin paddle (Fig. 4).

Postoperatively, the patient developed venous thrombosis of the flap after being administered desmopressin for idiopathic diabetes insipidus on day 5 [4]. Thrombectomy could be performed successfully and further healing process was uneventful. Postoperatively swallowing was possible very soon. The patient had a feeding tube for 14 days after surgery. After removal of it, she was able to eat a normal semi-solid diet and after wound healing (about 4 weeks postoperatively) also speech function recovered and verbal communication was possible, even though the speech was unclear. The patient also received an epithesis including the nasal and right orbital area and had an excellent functional and aesthetic result (Figs. 5, 7 and 8).

\section{Discussion}

Taylor et al. [1] first described the classic DCIA perforator flap and its use in facial (mandibular) reconstruction. The use has been limited because of the "unnecessary bulk of its obligatory muscle cuff" and the tethering of the skin to the bone. Safak et al. [5] and Kimata et al. [6] tried to reduce the adjacent soft tissue of the flap and achieved good results. Safak et al. [5] reported in 1997 that the skin paddle of the DCIA flap is supplied in $30 \%$ of the cases by a dominant cutaneous branch of the DCIA. The authors concluded that the adjacent soft tissue, which had been routinely harvested together with the skin to ensure adequate perfusion, was not necessary in these cases. Soft

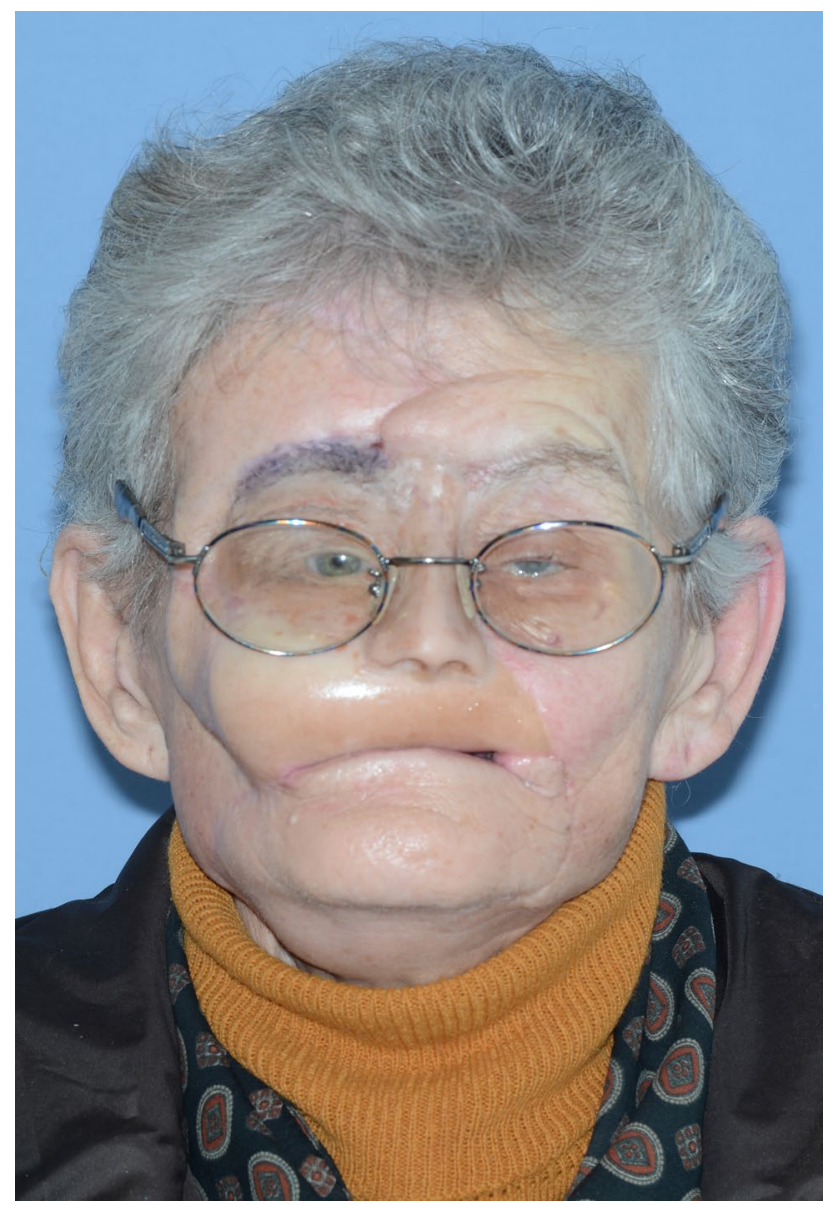

Fig. 5 The patient several weeks after surgery, wearing her epithesis of the nose and right eye

tissue bulk could thus be reduced and the problem of skin tethering to the bone could be overcome.

Akuyrek et al. [7] identified a long DCIA perforator vessel in 10 of the 12 cases in a cadaveric study and described an anatomical situation quite similar to the one we observed in our patient. The authors raised the flap without the iliac crest and described its use for local treatment of urogenital defects and its use as a free flap for covering a chronic wound on the lower leg, but they did not consider using the flap for the treatment of facial defects.

In the case of our patient, it was necessary to raise a flap with a bony part due to the large maxillectomy defect. "Bulkiness", a disadvantage often mentioned in connection with a DCIAP flap, was not a problem; on the contrary, we required a large volume of adjacent soft tissue because of the extensive defect.

A few years ago, Shaw and Brown [8] demonstrated the high value of DCIA perforator flaps for facial reconstruction. One of the advantages they mentioned was the skin pigmentation, which, although in fact not ideal, is much better than that in forearm or scapula flaps. The groin skin is also relatively hairless. Our DCIAP flap variant is in complete agreement with the description of their anatomic observation. They reported that either one or two 
Fig. 6 Schematic illustration of the intraoperative anatomical situation of the groin region in this unique case

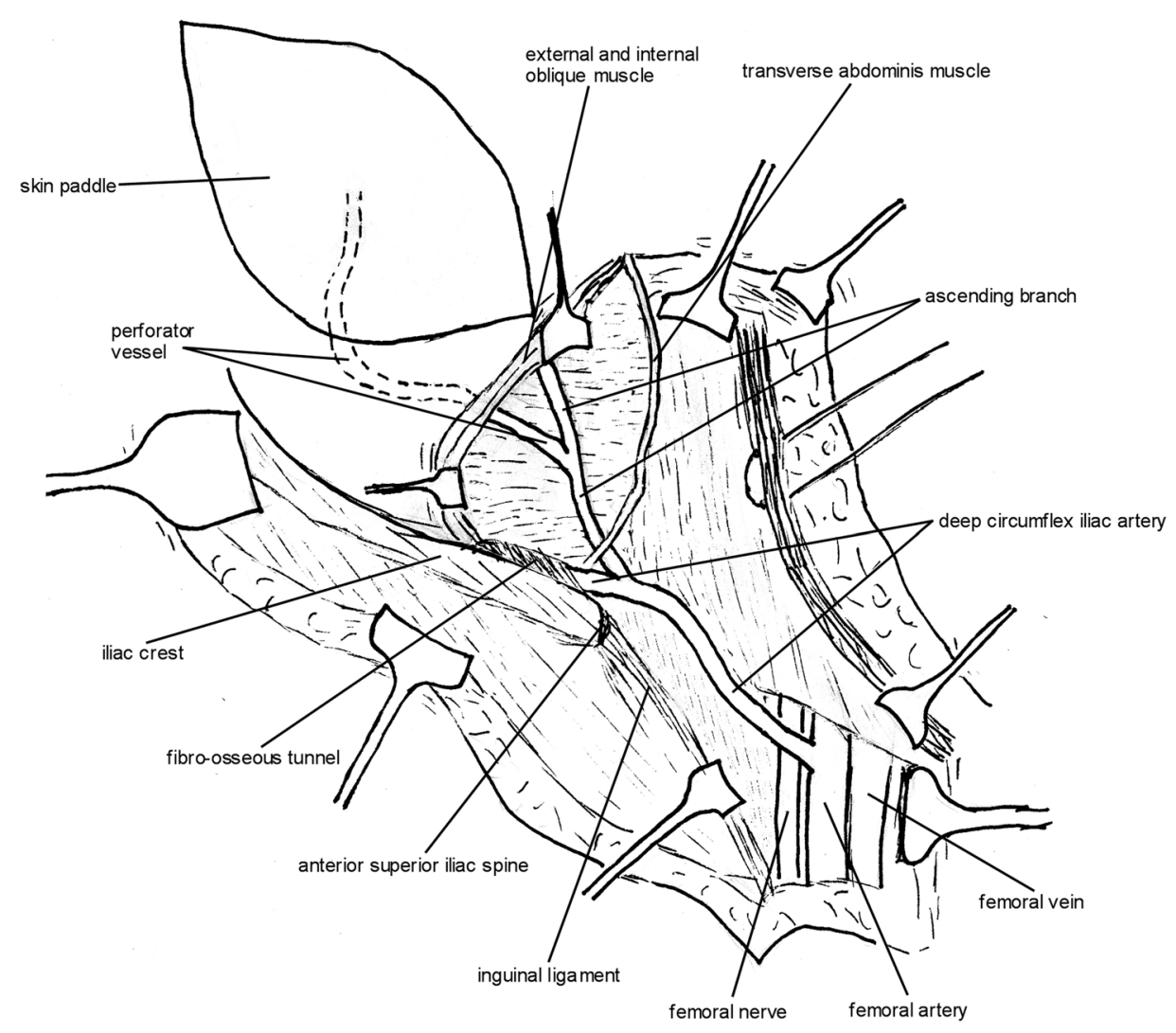

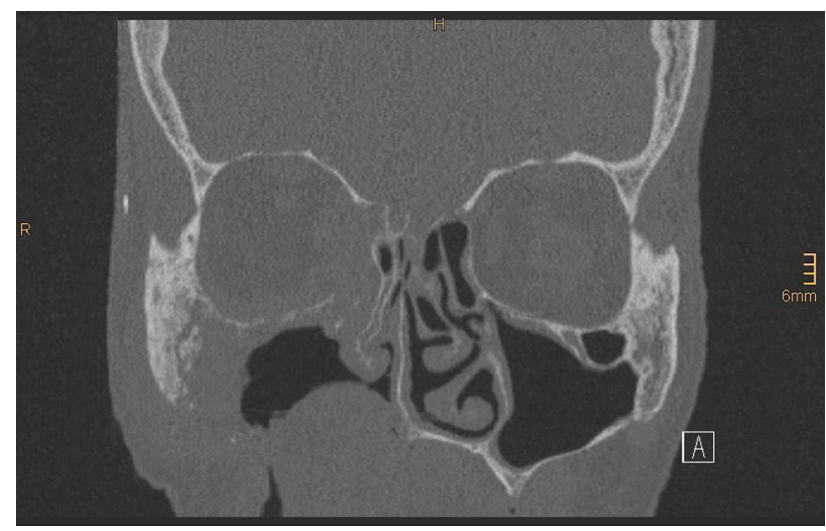

Fig. 7 Preoperative computer tomography scan showing complete destruction of the right maxilla and hard palate and bony infiltration of the orbital floor, zygomatic bone and ethmoidal cells

perforators originate from the ascending branch of the DCIA 5-11 cm posterior to the ASIS. They did not raise a flap larger than $6 \times 8 \mathrm{~cm}$ centered on these perforators but found that the vascularity was excellent.

In 2009, Ting et al. [9] evaluated 44 hemiabdominal walls with computed tomography angiography (CTA) before DCIA flap surgery. They observed high variability and concluded that exact preoperative evaluation is only possible with CTA, but that the osteomusculocutanous DCIAP flap is a good option for facial reconstruction in general. Bergeron et al. [10] also performed an anatomical study of the groin region and found perforators origi-

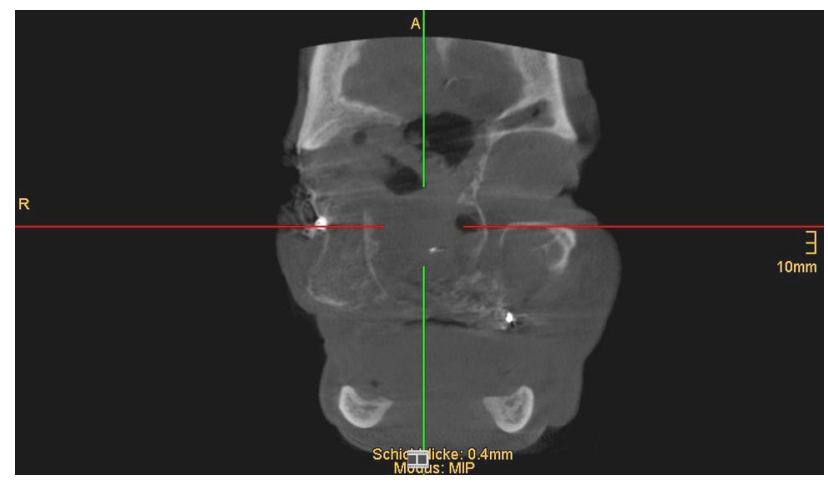

Fig. 8 Postoperative computer tomography scan showing the bony reconstruction of the zygomatic contour and the maxilla and partial reconstruction of the bony orbit

nating from the DCIA in $92 \%$ of the cases investigated. They also recommended preoperative CTA. Although we did not use CTA, because ultrasound confirmed the availability of the standard DCIA anatomy, we would recommend doing it preoperatively for exact surgical planning of raising the best flap available in the unique anatomical situation of each patient, as we have learned in this case.

Ting et al. [11] recently provided an interesting approach for individual DCIA flap harvest. The authors also strengthen the importance of preoperative CTA and also introduce advanced imaging modalities and other technologies such as image-guided stereolithographic biomodeling for preoperative flap planning. 


\section{Conclusion}

The observed anatomy in our patient has already been described, but such a DCIAP free flap has never been raised and reported in literature before (large skin paddle, long vascular pedicles, and osteomuscular part). It allows high mobility and is thus an excellent option for facial reconstruction. The literature analysis also revealed that nowadays preoperative CTA or MRA should be standard for a DCIAP flap harvest (Figs. 1, 2, 3, 4, 5, 6, 7 and 8).

\section{Ethical approval}

Not required.

\section{Photo documents}

Permission for publication obtained.

\section{Conflict of interest}

The authors have no financial or personal relationships with other people or organizations that could inappropriately influence (bias) this work.

No funding was received for this work. None of the authors has a financial interest in any of the products, devices, or drugs mentioned in this manuscript.

\section{References}

1. Taylor GI, Townsend P, Corlett R. Superiority of the deep circumflex iliac vessels as the supply for free groin flaps: clinical work. Plast Reconstr Surg. 1979;64:745.

2. Taylor GI, Townsend P, Corlett R. Superiority of the deep circumflex iliac vessels as the supply for free groin flaps. Experimental work. Plast Reconstr Surg. 1979;64(5):595-604.
3. Koshima I, Yamamoto H, Hosoda M, Moriguchi T, Orita Y, Nagayama $\mathrm{H}$. Free combined composite flaps using the lateral circumflex femoral system for repair of massive defects of the head and neck regions: an introduction to the chimeric flap principle. Plast Reconstr Surg. 1993;92(3):411-20.

4. Ensat F, Wakolbinger R, Schubert HM, Moser G, Hladik M, Wechselberger G. Venous thrombosis of free flap immediately after intravenous application of desmopressin. Microsurgery. 2013;33(1):79-80.

5. Safak T, Klebuc MJ, Mavili E, Shenaq SM. A new design of the iliac crest microsurgical free flap without including the "obligatory" muscle cuff. Plast Reconstr Surg. 1997;100(7):1703-9.

6. Kimata Y, Uchiyama K, Sakuraba M, Ebihara S, Hayashi R, Asakage T, Nakatsuka T, Harii K. Deep circumflex iliac perforator flap with iliac crest for mandibular reconstruction. Br J Plast Surg. 2001;54(6):487-90.

7. Akyurek M, Conejero A, Dunn R. Deep circumflex iliac artery perforator flap without iliac crest. Plast Reconstr Surg. 2008;122(6):1790-5.

8. Shaw JR, Brown JS. Osteomyocutanous deep circumflex iliac artery perforator flap in the reconstruction of midface defect with facial skin loss: a case report. Microsurgery. 2009;29:299-302.

9. Ting JW, Rozen WM, Grinsell D, Stella DL, Ashton MW. The in vivo anatomy of the deep circumflex iliac artery perforators: defining the role for the DCIA perforator flap. Microsurgery. 2009;29(4):326-9.

10. Bergeron L, Tang M, Morris SF. The anatomical basis of the deep circumflex iliac artery perforator flap with iliac crest. Plast Reconstr Surg. 2007;120(1):252-8.

11. Ting JW, Rozen WM, Niumsawatt V, Baillieu C, Leung M, Leong JC. Developments in image-guided deep circumflex iliac artery flap harvest: a step-by-step guide and literature review. J Oral Maxillofac Surg. 2014;72(1):186-97. 